\section{A convenient aligner solution}

Henry Schein Dental has launched Reveal Clear Aligners (Reveal), a convenient solution for patients looking to enhance their smile, with a treatment plan approved by a dentist of their choice. Designed with patient comfort and aesthetics in mind, the aligners are clearer than most other aligners in the market and reduce or eliminate the need for attachments in mild to moderate cases, featuring a smooth scalloped edge that enhances patient comfort.

For general practitioners, Reveal easily integrates into the dental practice workflow. Henry Schein's Orthodontic Specialists provide in-practice support of the system and advice on how to incorporate aligner workflows into your treatment plans.

Reveal cases are also transmitted within Software of Excellence's Digital Dental Exchange (DDX), a web-based portal that enables practitioners to submit, review, and track aligner cases. Dental professionals can upload digital impression images or submit traditional impressions if they prefer. Once all patient information is uploaded, the Reveal lab will generate a recommended treatment plan promptly for the practitioner to approve. The practitioner reviews and approves the treatment, then tracks the progress of the case and makes any necessary adjustments.

For more information, visit www.henryschein.co.uk.

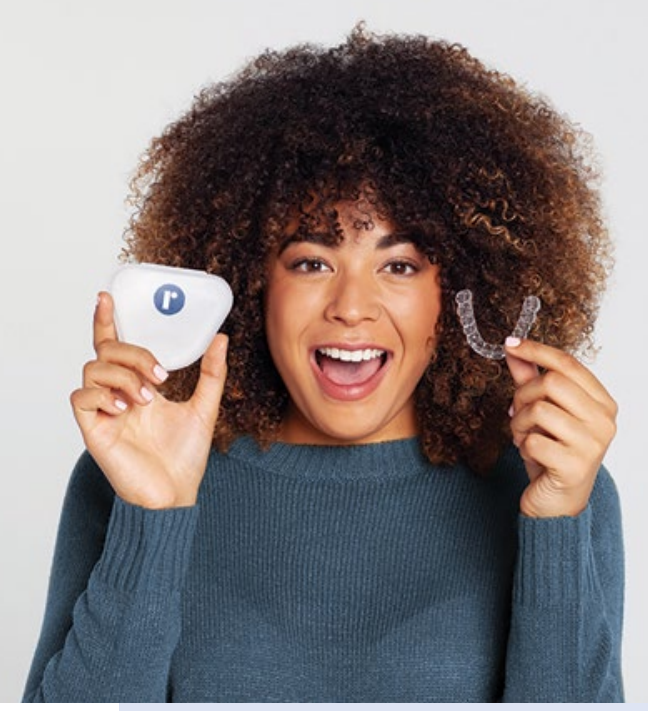

\section{Your first line of defence}

Are you worried about what to do should you have a suspected case of coronavirus in your aesthetic clinic?

Put your fears at ease with the new Infection Containment Kit from Initial Medical, designed for touchpoint cleaning and the disinfection of potentially contaminated surfaces.

Containing PPE, specialist Steri-7 infection control products, hand sanitiser, workplace barrier tape and a rigid container for waste disposal, this kit is the best first response you can have to hand for a suspected infection onsite. The kit comes with complete stepby-step guidelines on how to use each of the items inside, and once used the waste will be collected and disposed of responsibly by the Initial Medical team.

Suitable to help contain a wide range of suspected infections including coronavirus, norovirus, MRSA and many more, it's the perfect solution to have on hand. In the event of a confirmed case of coronavirus onsite then a specialist disinfection service should be undertaken by professionals such as Rentokil Specialist Hygiene who are equipped with the relevant correct personal protective equipment (PPE), respiratory protective equipment (RPE) and specialist disinfection equipment.

For further information visit www.initial. co.uk/medical or call 08708504045 .

\title{
Pre-loading face mask dispenser
}

Developed in conjunction with a number of NHS Trusts, the SaniStation range is specifically designed to provide infection control to protect your patients, staff and visitors from cross-contamination, and is brought to you by a group who played a key role in establishing the Nightingale Centres. The units start from just $£ 98$.

The unique design, registered face mask dispenser can be located at your practice entrance, ensuring all patients have access to appropriate and hygienic face coverings. With a clever pre-loader mechanism, the lockable, $250+$ capacity unit is quick and simple to fill, leaving your staff free to concentrate on patient care.

The mask

dispenser can either be wall-mounted or combined with a robust, free-standing, mobile sanitisation station, which can include bespoke options such as a touchless automatic hand gel dispenser, apron dispenser, glove dispenser and touchless bin.

The stations can be branded with your practice logo and hygiene instructions and positioned at your practice entrance and/ or exit. A large number of NHS Trusts, private hospitals and clinics across GB and NI have already chosen SaniStation for their transmission prevention, so you can be assured that they meet the very highest safety standards (https://sani-station.co.uk/ nhs-sanistation).

Dentists' Provident members received a $10 \%$ discount. Contact Shane O'Byrne, New Business Manager for more details on 07554861403 or shane.obyrne@sanistation.co.uk. 